Статья с открытым доступом под лицензией CC BY-NC-ND (https://creativecommons.org/licenses/by-nc-nd/3.0/) Материалы Международной практической интернет-конференции «Актуальные проблемы науки»

Выпуск II, ноябрь 2019

ISBN 978-601-323-144-0

https://doi.org/10.31643/2019.024

\author{
Мендакулов Жасулан Корабаевич \\ Satbayev University, Казахстан \\ E-mail: m_m_zhas@mail.ru \\ OORCID ID: 0000-0002-3818-404X
}

\title{
Улучшение точности позиционирования в закрытых помещениях используя данные экспериментальных измерений и корректировки многочастотным методом
}

\begin{abstract}
Абстракт: Определение местоположения объекта в закрытых помещениях можно осуществить применяя теоретические данные. Теоретические исследования определяют координаты объекта, но в практических приложениях координаты объекта определяются с ошибками. Это происходит из-за наличия шумов в окружающем пространстве. Необходимо провести экспериментальные исследования, определяющие практические значения показаний уровней мощности от маяков. Применяя эти данные, можно скорректировать точность координат искомого объекта. Эксперимент проводится в трех конфигурациях: а) Измерение уровней мощности при расположении объектов эксперимента: передатчика и приемника, при прямой видимости; b) Измерение уровней мощности при расположении объектов эксперимента: передатчика и приемника, при не прямой видимости. Объект преграды - метал; с) Измерение уровней мощности при расположении объектов эксперимента: передатчика и приемника, при не прямой видимости. Объект преграды - стена. Результаты эксперимента могут быть использованы для корректировки теоретических данных. Применяя, многочастотный передатчик и приемник, можно улучшить точность позиционирования.

Ключевые слова: line-of-sight, non-line-of-sight, анализатор спектра, USB-генератор.
\end{abstract}

\section{Введение}

Необходимое оборудование для проведения эксперимента:

a) Анализатор спектра БАРС. Внешний вид анализатора спектра представлен на рисунке 1.

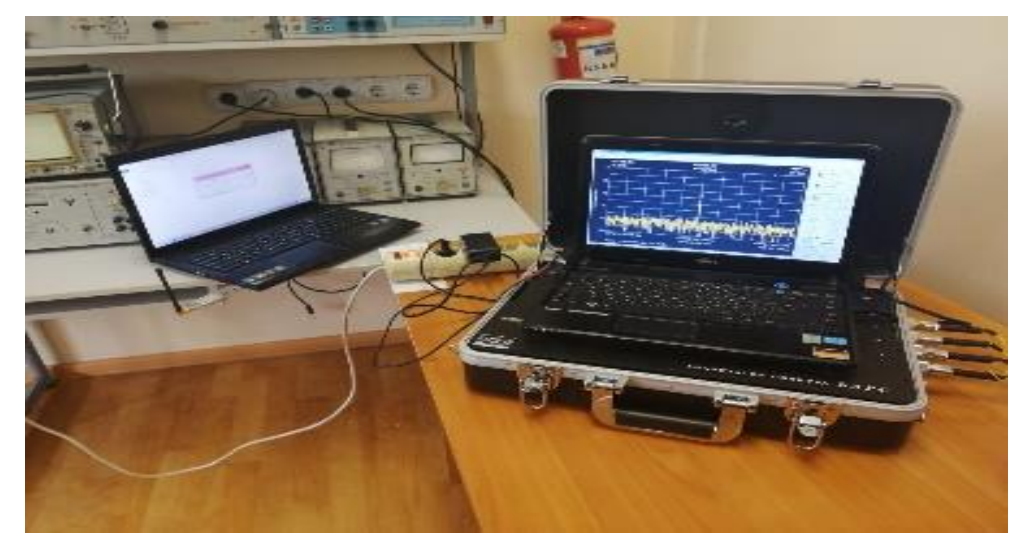

Рисунок 1 - Пример измерения уровня мощности сигнала частоты 2400 МГц от USB-генератора на анализаторе спектра БАРС 
Внешний вид модуля анализатора спектра представлен на рисунке 2.

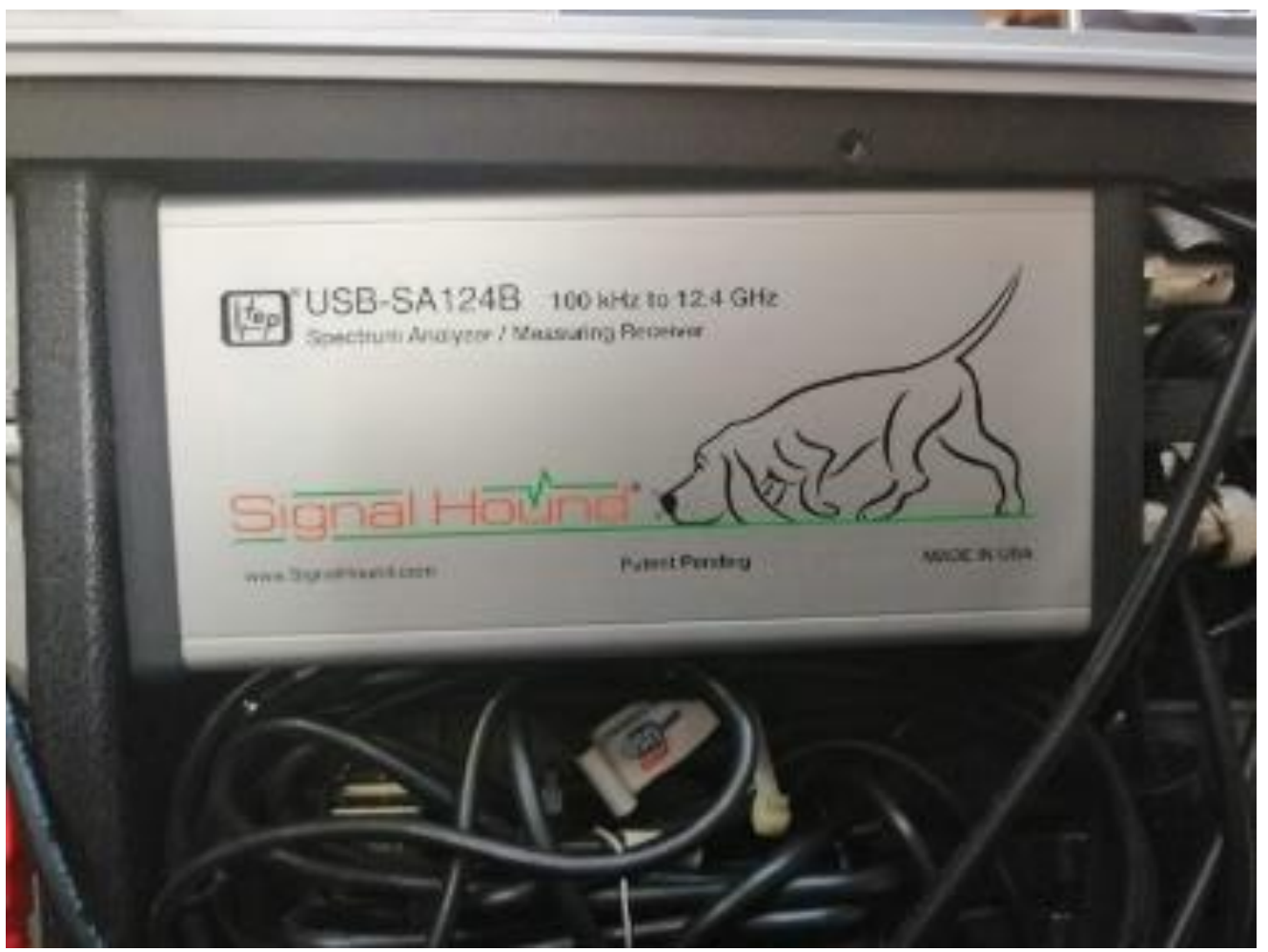

Рисунок 2 - Модуль анализатора спектра USB-SA124B (100 кГц-12.4 ГГц)

Внешний вид окна настроек анализатора спектра БАРС представлен на рисунке 3.

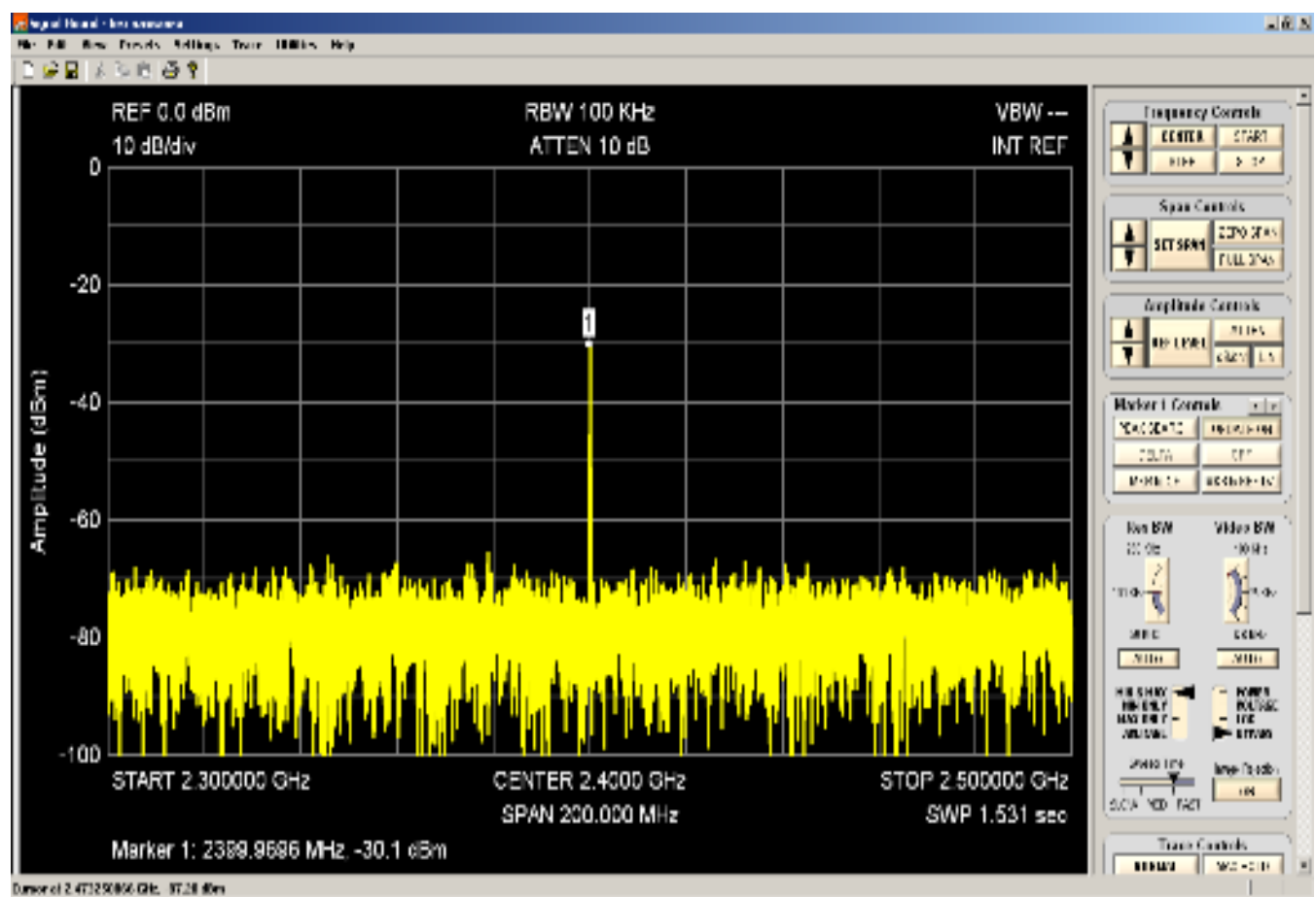

Рисунок 3 - Основное окно программы для задания частоты измерения и настроек

Технические характеристики анализатора спектра БАРС: диапазон частот 1 Гц-4.4 (12.4) ГГц; динамический диапазон (-151)-(+10) дБм; разрешение по частоте (RBW) 0.1 Гц-250 кГц; точность 
измерения уровней 0.25 дБ; полоса частот I/Q демодулятора 240 кГц; скорость сканирования 140 МГц/с; количество каналов 5 .

b) USB-генератор. Технические характеристики: рабочая полоса частот от 25 Гц-до 6 ГГц. Окно задания частоты, ослабления и режима USB генератора представлен на рисунке 4.

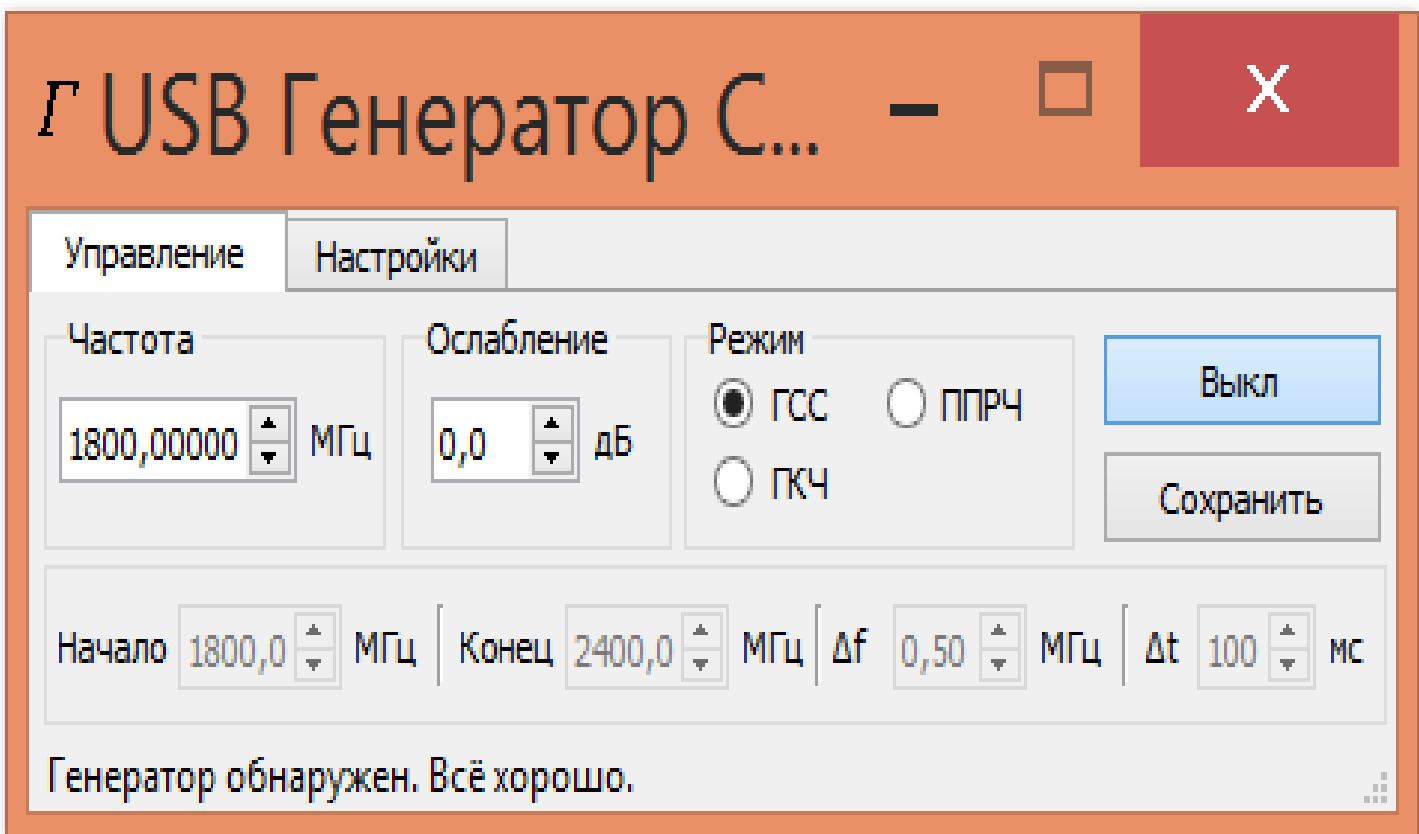

Рисунок 4 - Окно задания частоты, ослабления и режима USB генератора

Внешний вид USB-генератора представлен на рисунке 5.

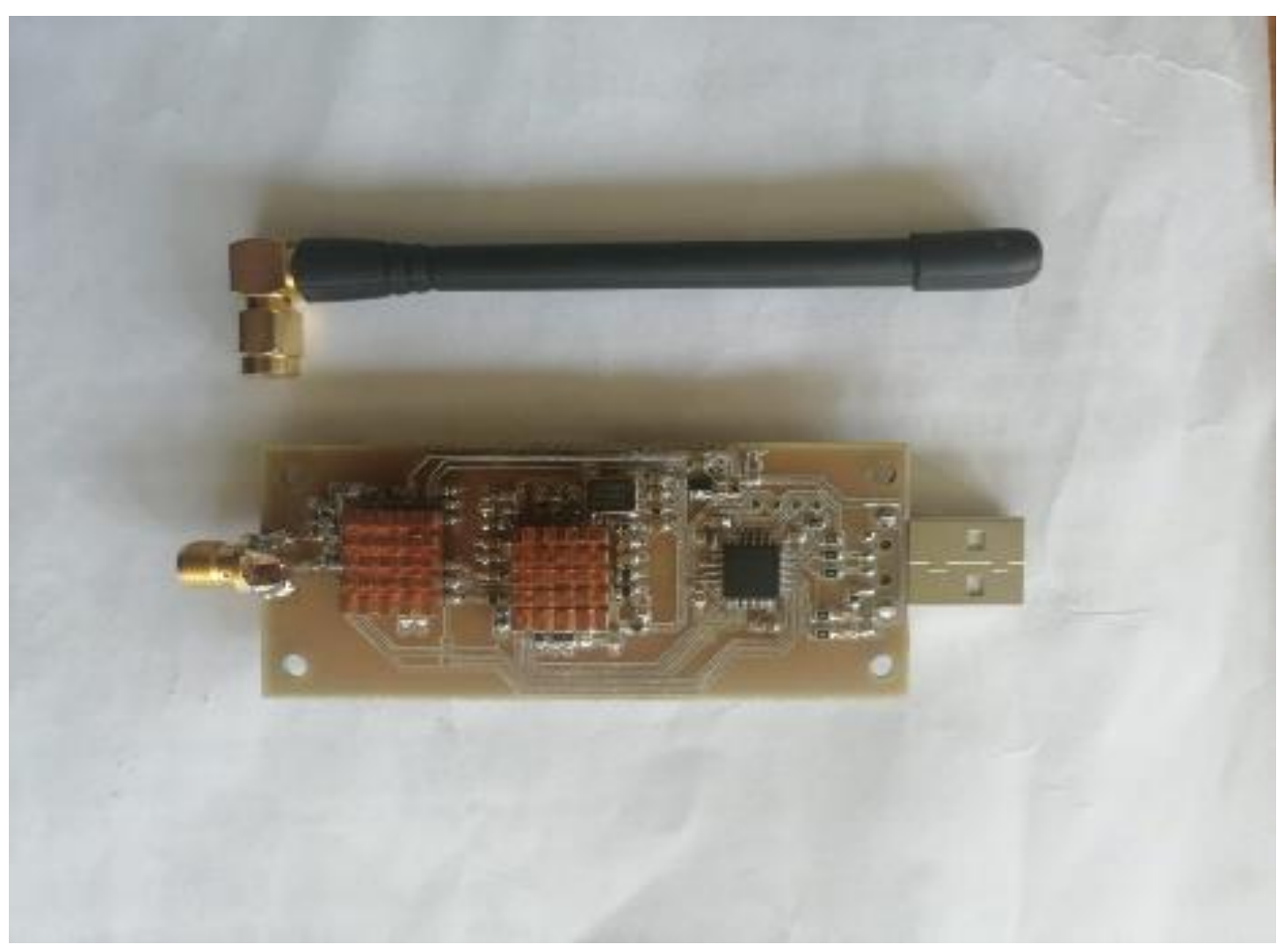

Рисунок 5 - Внешний вид USB-генератора и его антенны 


\section{Описание эксперимента}

1) Измерение уровня мощности сигнала в зависимости от расстояния при прямой видимости (line-of-sight (LOS)) в закрытом помещении. Структурная схема эксперимента приведена на рисунке 6.

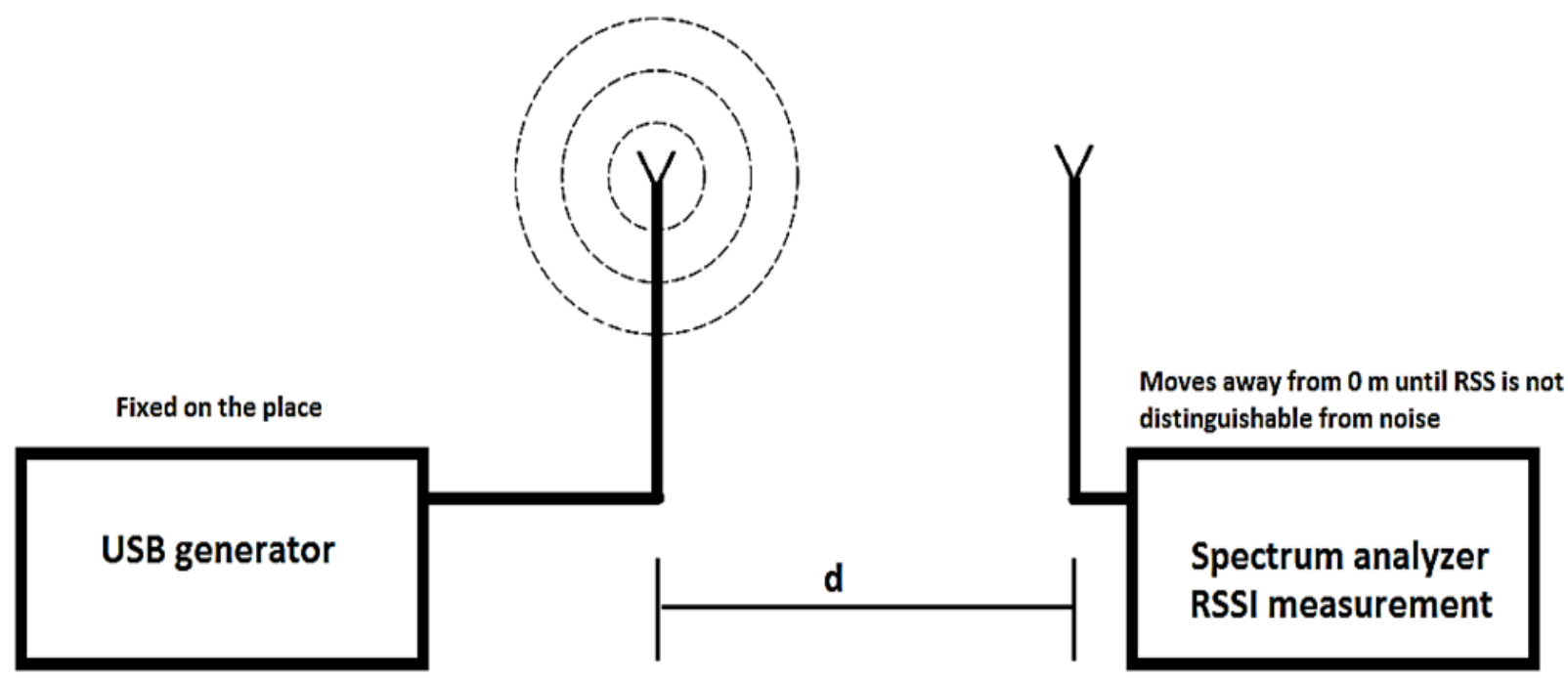

Рисунок 6 - Расположение объектов эксперимента для измерения при прямой видимости

Генератор фиксируется на месте с известными координатами. Задается частота вещания. Приемник фиксируется возле генератора. Затем приемник отодвигается от генератора на 1 метр. Фиксируется значение показания уровня мощности на анализаторе спектра. Эксперимент проводится до того расстояния, пока уровень мощности не будет различим от уровня мощности шума. На приемнике устанавливается антенна соответствующей длины. Длина антенны выбирается соответствующей длине волны синусоиды. Проведение измерений при частотах 1800 МГц, 2000 МГц, 2200 МГц и 2400 МГц. График представлен на рисунке 7.

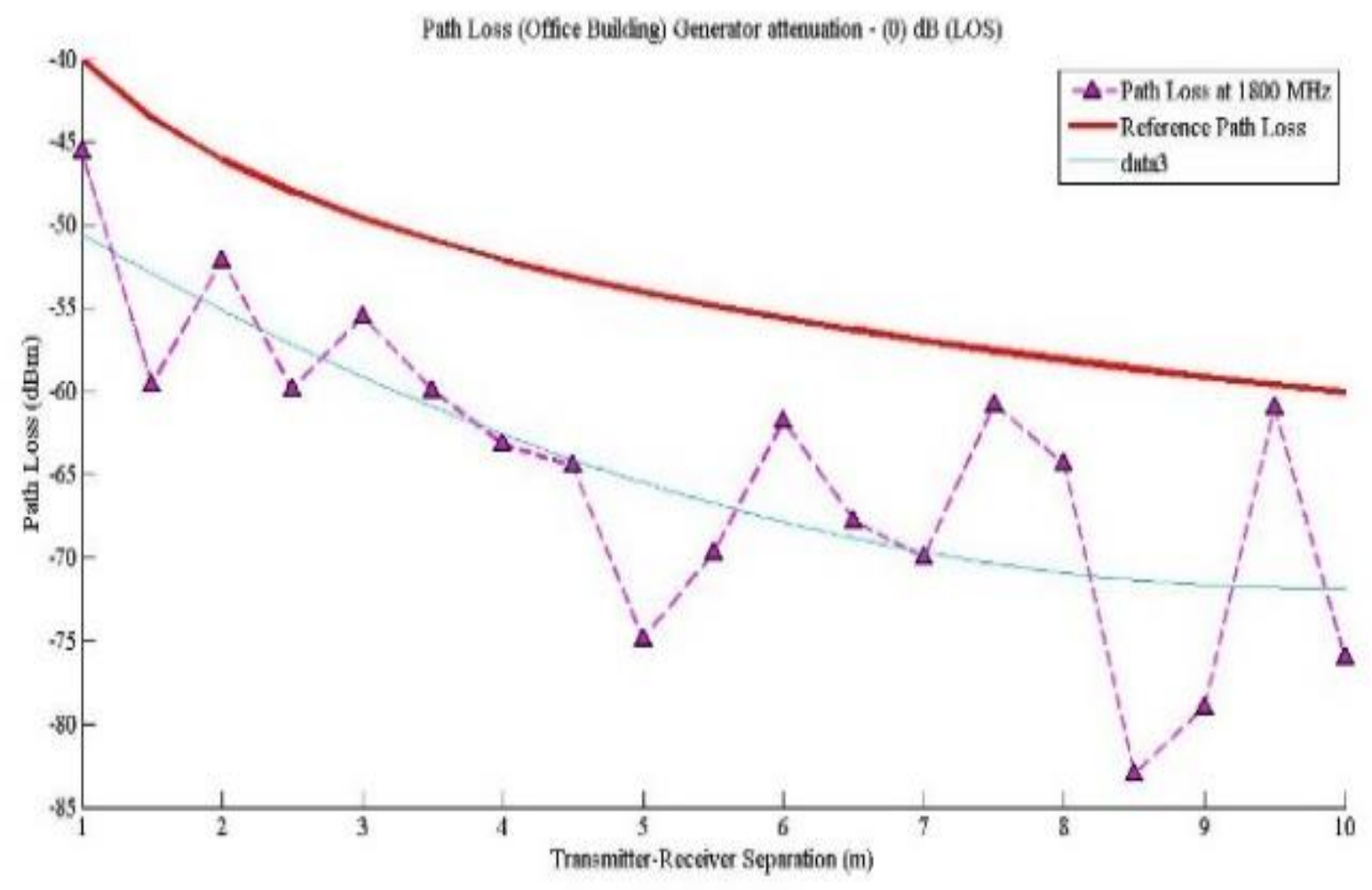



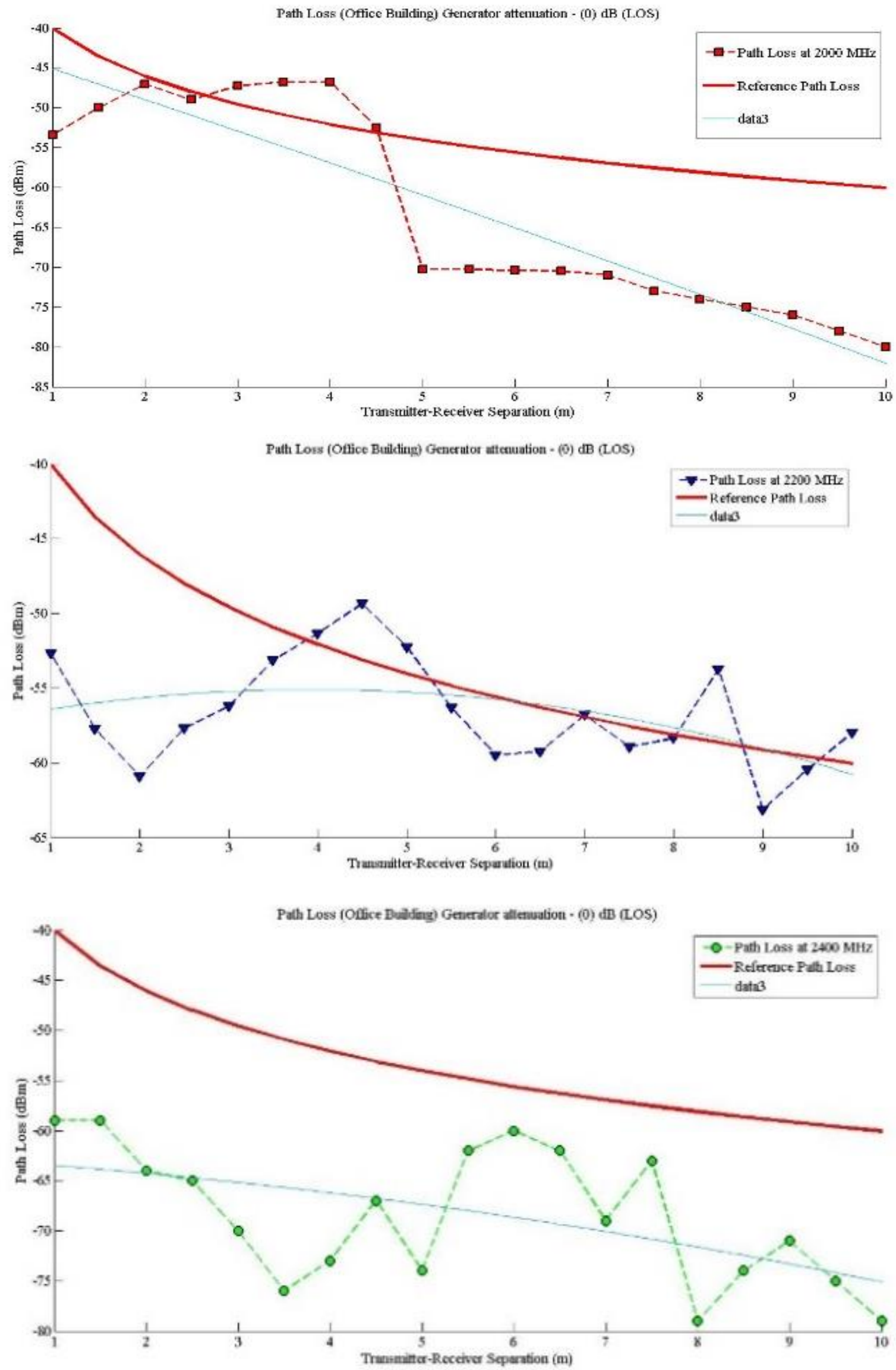

Рисунок 7 - Зависимость уровня мощности сигнала в зависимости от расстояния при прямой видимости при частотах 1800 МГц, 2000 МГц, 2200 МГц и 2400 МГц (офисное помещение) 
2) Измерение уровня мощности сигнала в зависимости от расстояния при не прямой видимости (non-line-of-sight (NLOS)) в закрытом помещении. Материал преграды - железо. Структурная схема эксперимента приведена на рисунке 8.

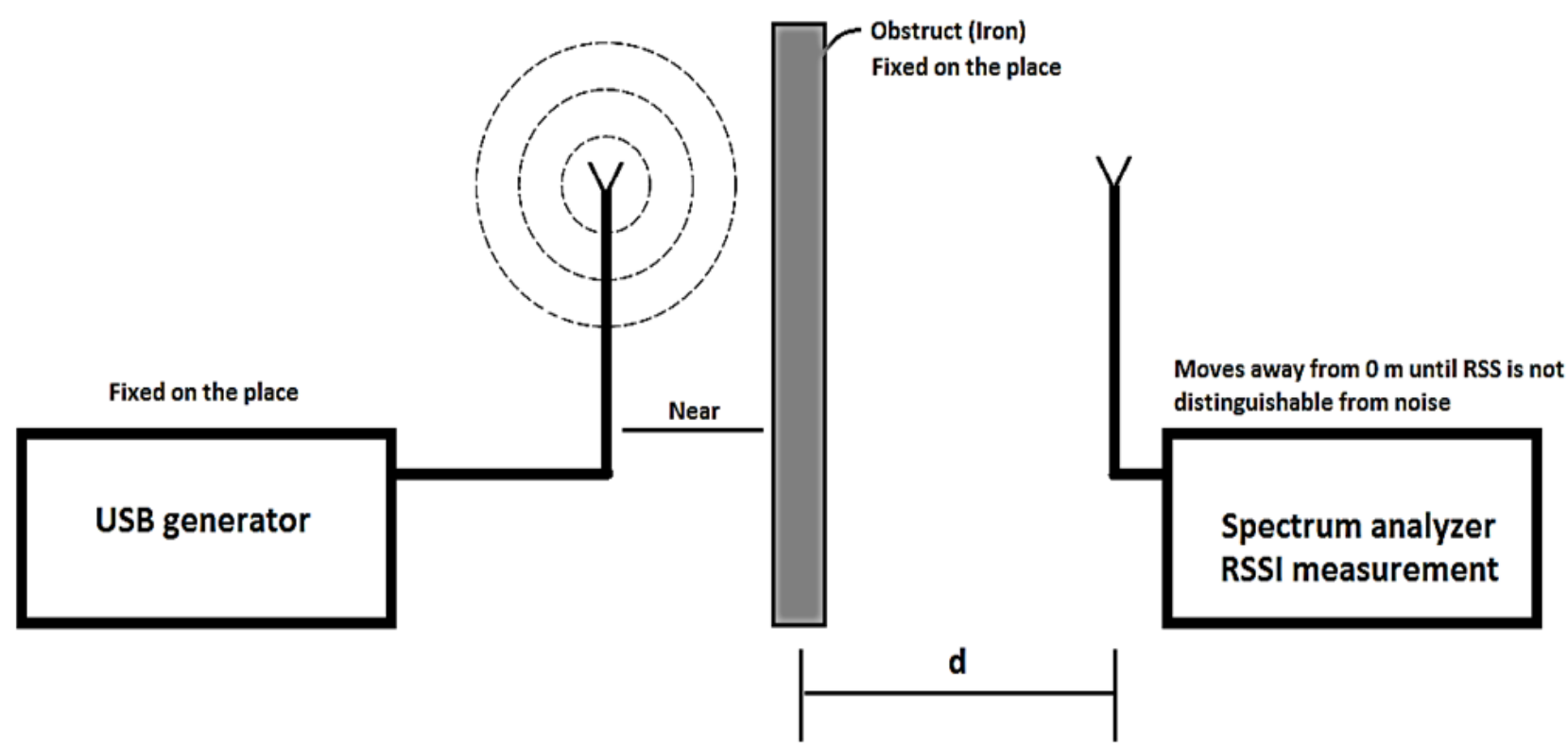

Рисунок 8 - Расположение объектов эксперимента для измерения при не прямой видимости. Материал преграды - железо

Генератор фиксируется на месте с известными координатами. Задается частота вещания. Приемник фиксируется возле генератора. Затем приемник отодвигается от генератора на 1 метр. Между ними устанавливается преграда из железа. Преграда имеет прямоугольную форму. Она не окружает генератор, а лишь закрывает часть пространства между генератором и приемником. Фиксируется значение показания уровня мощности на анализаторе спектра. Эксперимент проводится до того расстояния, пока уровень мощности не будет различим от уровня мощности шума. Преграда во все время эксперимента фиксируется на одном и том же месте. На приемнике устанавливается антенна соответствующей длины. Длина антенны выбирается соответствующей длине волны синусоиды.

a) Проведение измерений при частотах 1800 МГц, 2000 МГЦ, 2200 МГц и 2400 МГц.

График представлен на рисунке 9.

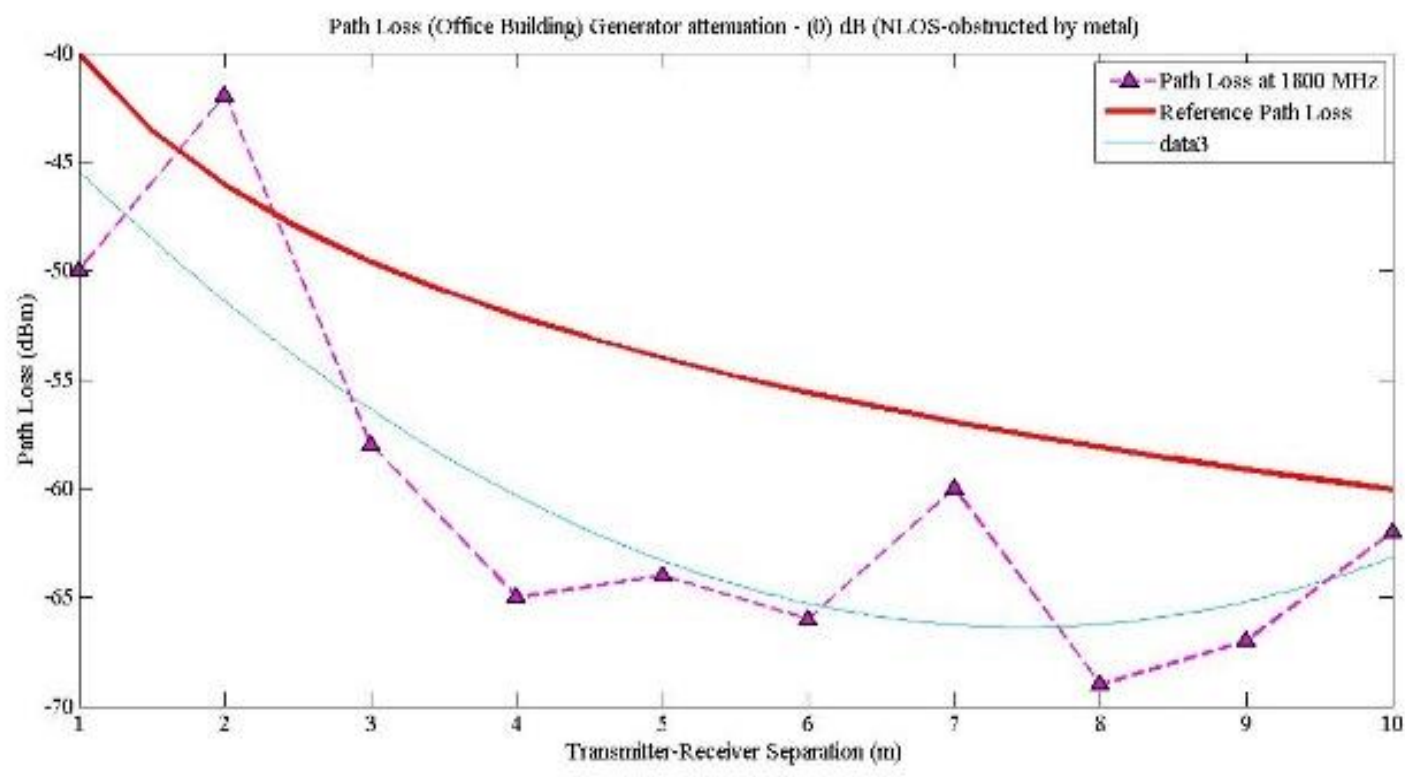



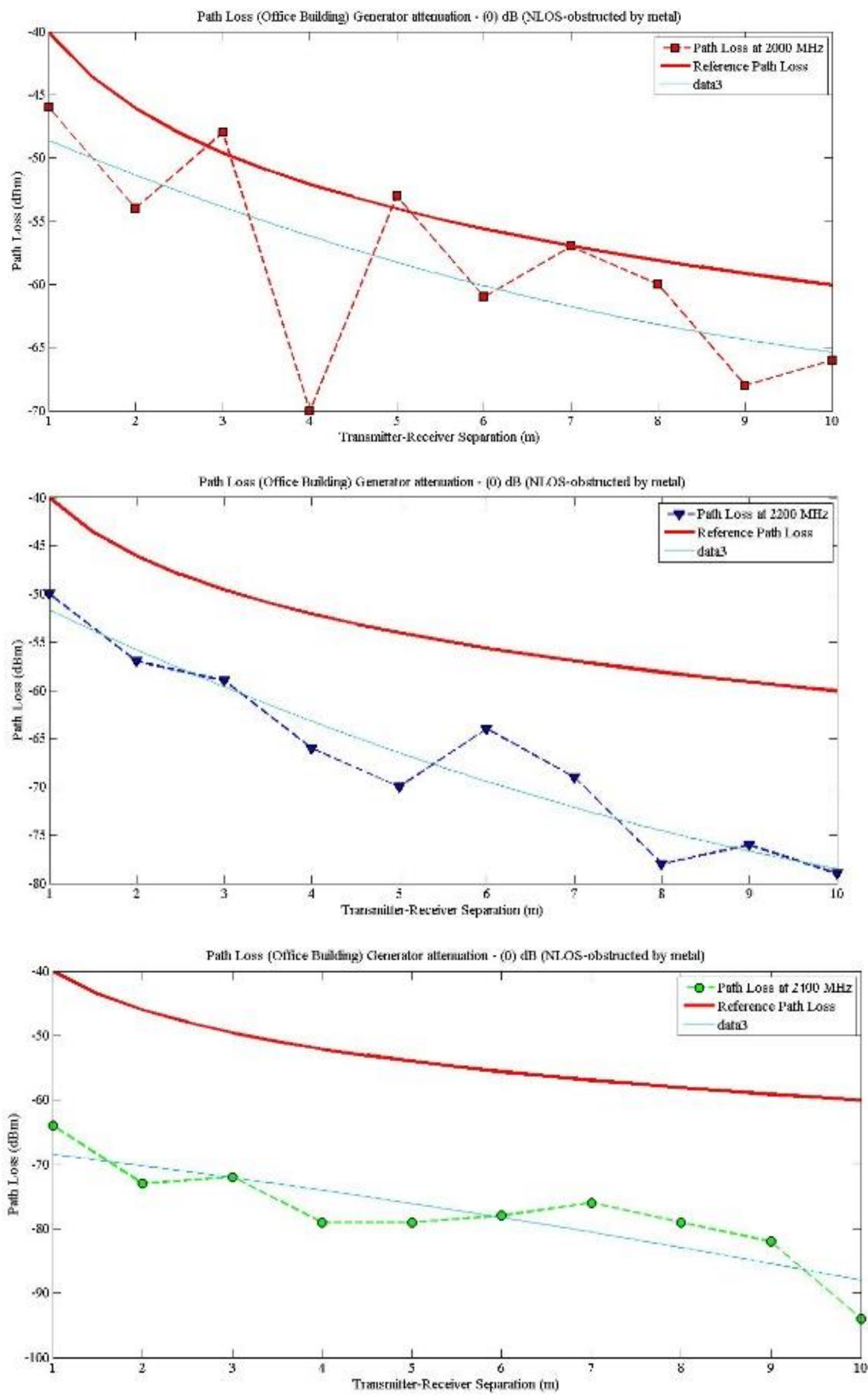

Рисунок 9 - Зависимость уровня мощности сигнала в зависимости от расстояния при не прямой видимости при частотах 1800 МГц, 2000 МГц, 2200 МГц и 2400 МГц (офисное помещение). Материал преграды - железо 
3) Измерение уровня мощности сигнала в зависимости от расстояния при не прямой видимости (non-line-of-sight (NLOS)) в закрытом помещении. Материал преграды - стена. Структурная схема эксперимента приведена на рисунке 10.

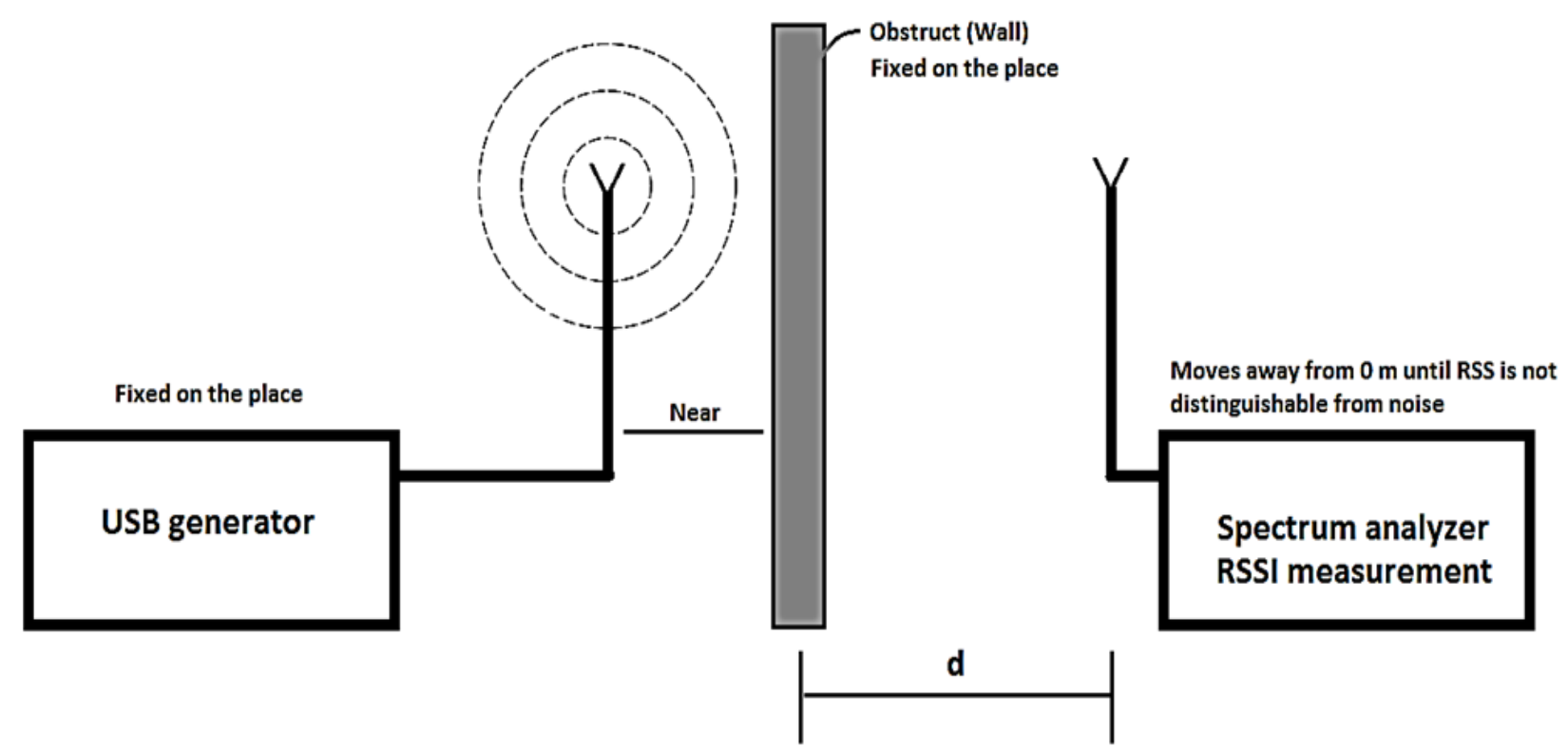

Рисунок 10 - Расположение объектов эксперимента для измерения при не прямой видимости. Материал преграды - стена

Генератор фиксируется на месте с известными координатами. Задается частота вещания. Приемник фиксируется возле генератора. Затем приемник отодвигается от генератора на 1 метр. Между ними устанавливается преграда в виде стены. Генератор находился в одной комнате, а приемник располагается за стеной. Фиксируется значение показания уровня мощности на анализаторе спектра. Эксперимент проводится до того расстояния, пока уровень мощности не будет различим от уровня мощности шума. Генератор во все время эксперимента находится на одном и том же месте. На приемнике устанавливается антенна соответствующей длины. Длина антенны выбирается соответствующей длине волны синусоиды.

a) Проведение измерений при частотах 1800 МГц, 2000 МГц, 2200 МГц и 2400 МГц. График представлен на рисунке 11.

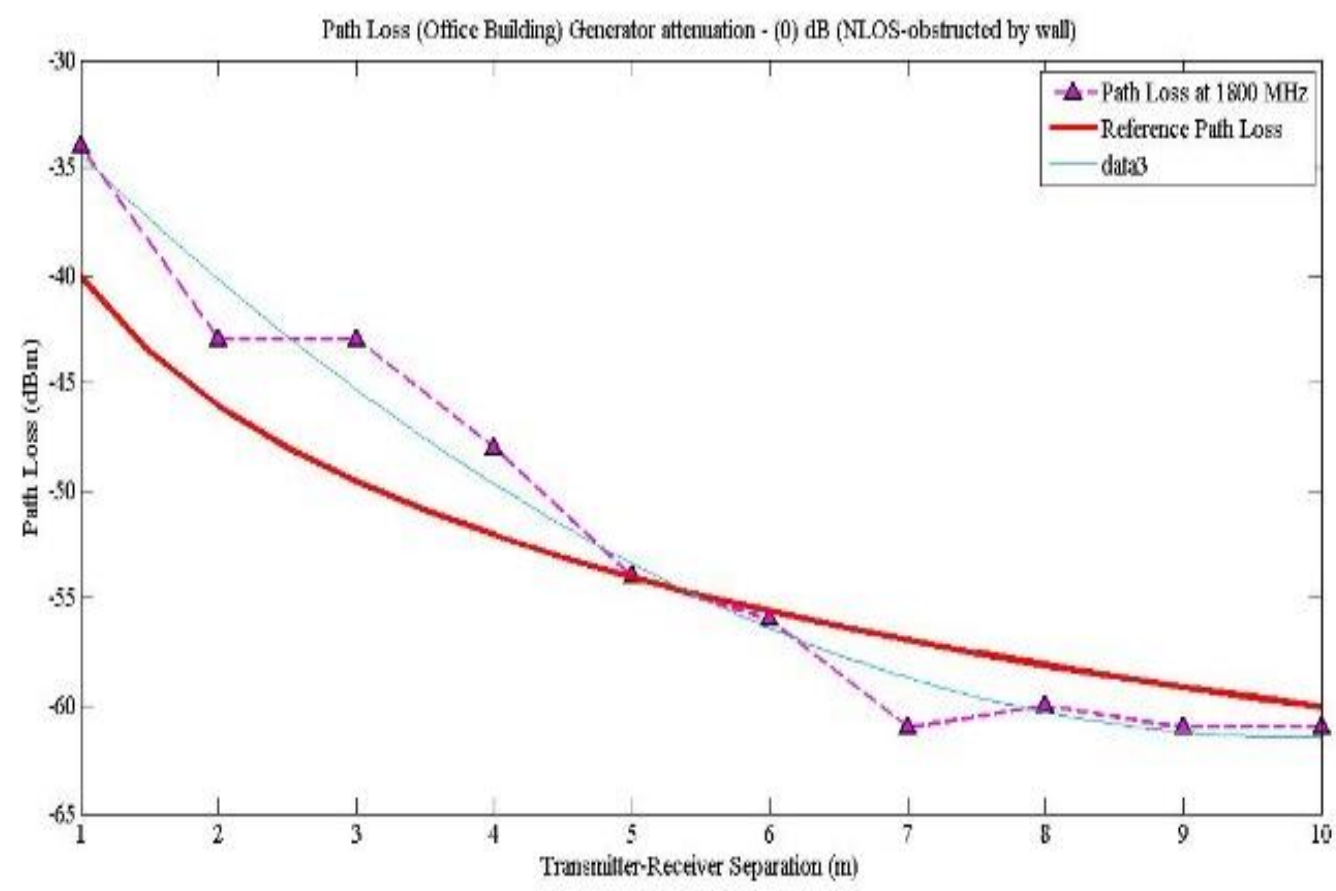



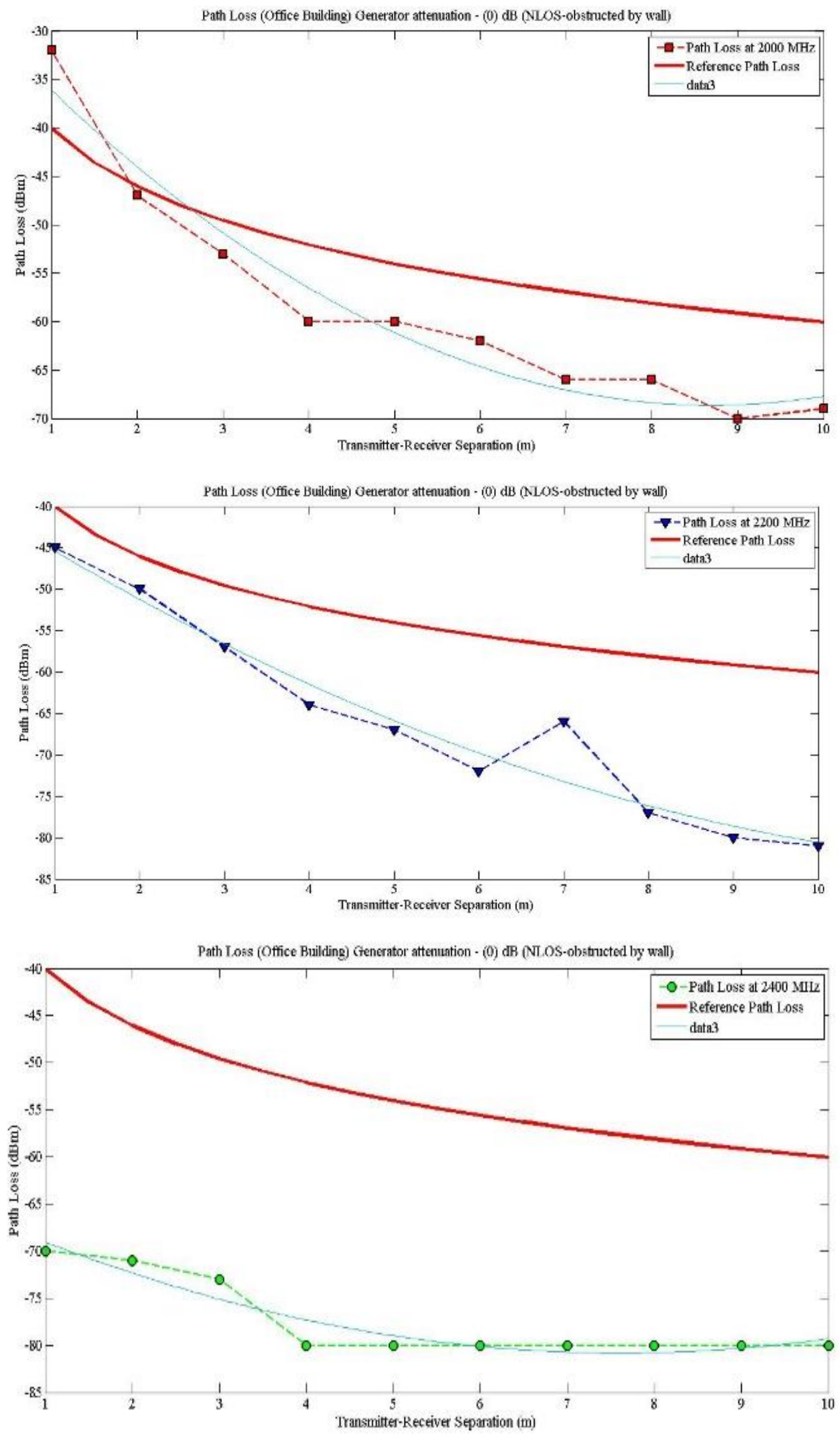

Рисунок 11 - Зависимость уровня мощности сигнала в зависимости от расстояния при не прямой видимости при частотах 1800 МГц, 2000 МГц, 2200 МГц и 2400 МГц (офисное помещение). Материал преграды - стена 


\title{
Заключение
}

Применяя, полученные практические данные можно скорректировать точность данных позиционирования, полученных теоретическими методами. Интерполированные кривые на каждой частоте показывают различное приближение к эталонной кривой. Если передатчик настроить на многочастотный режим передачи и использовать среднюю арифметическую всех кривых можно учесть ошибки каждой частоты и повысить точность.

\author{
Mendakulov Zhassulan Korabaevich \\ Satbayev University, Kazakhstan \\ E-mail: m_m_zhas@mail.ru \\ 0ORCID ID: 0000-0002-3818-404X
}

\section{Improvement of positioning accuracy in closed rooms by using of experimental measurement data and correction of multifrequency method}

\begin{abstract}
Annotation. Determining of the location of an object in enclosed spaces can be done using theoretical data. But in practical situations coordinates of object are found with errors. It takes place because of presence of noise in environment. It is necessary to carry out experimental research to receive practical readings of power levels from beacons. It is possible to correct coordinates of searching object, that was taken theoretically by using these data. Experiment carried out in three configurations: a) measurement of power level when transmitter and receiver located line-of-sight; b) measurement of power level when transmitter and receiver located non-line-of-sight. Obstacle object - metal; c) measurement of power level when transmitter and receiver located non-line-of-sight. Obstacle object - wall.
\end{abstract}

Keywords: line-of-sight, non-line-of-sight, spectrum analyzer, USB-generator.

Ссылка на данную статью: Мендакулов Ж. К. (2019), Улучшение точности позиционирования в закрытых помещениях используя данные экспериментальных измерений и корректировки многочастотным методом. Материалы Международной практической интернет-конференции «Актуальные проблемы науки» / Materials of International Practical Internet Conference "Challenges of Science”. ISBN 978-601-323-1440. Выпуск II, 2019. Стр.: 118 - 127. https://doi.org/10.31643/2019.024

\section{Литература}

[1] Ferdous Hossain 1,*, Tan Kim Geok 1,*, Tharek Abd Rahman 2, Mohammad Nour Hindia 3, Kaharudin Dimyati 3 , Sharif Ahmed 1, C. P. Tso 1, Azlan Abdaziz 1, W. Lim 1, Azwan Mahmud 4, Tan Choo Peng 5, Chia Pao Liew 6 and Vinesh Thiruchelvam Electronics 2019, 8, 750

[2] Simone Morosi, Alessio Martinelli, Enrico Del Re Peer-to-peer cooperation for GPS positioning: cooperative GPS positioning. Article in international journal of satellite communications and networking, July 2016

[3] S. Deng, M. K. Samimi, T. S. Rappaport, ”28 GHz and 73 GHz Millimeter-Wave Indoor Propagation Measurements and Path Loss Models," accepted at the 2015 IEEE International Conference on Communications Workshop (ICC Workshop), 8-12 June, 2015. 